\title{
Development, specification, and diversity of callosal projection neurons
}

\section{Citation}

Fame, Ryann M., Jessica L. MacDonald, and Jeffrey D. Macklis. 2011. “Development, specification, and diversity of callosal projection neurons." Trends in Neurosciences 34 (1) (January): 41-50.

\section{Published Version}

doi:10.1016/j.tins.2010.10.002

\section{Permanent link}

http://nrs.harvard.edu/urn-3:HUL.InstRepos:11130516

\section{Terms of Use}

This article was downloaded from Harvard University's DASH repository, and is made available under the terms and conditions applicable to Open Access Policy Articles, as set forth at http:// nrs.harvard.edu/urn-3:HUL.InstRepos:dash.current.terms-of-use\#OAP

\section{Share Your Story}

The Harvard community has made this article openly available.

Please share how this access benefits you. Submit a story.

Accessibility 


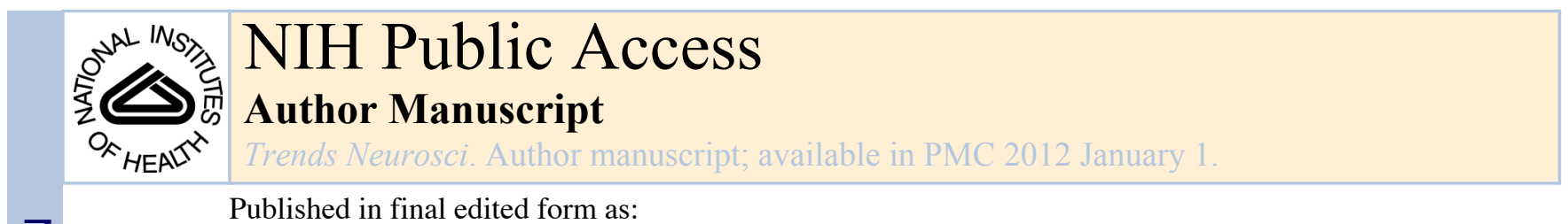

Trends Neurosci. 2011 January ; 34(1): 41-50. doi:10.1016/j.tins.2010.10.002.

\title{
Development, Specification, and Diversity of Callosal Projection
}

\section{Neurons}

\author{
Ryann M. Fame ${ }^{1,{ }^{*}, \text { Jessica L. MacDonald }}{ }^{1,}{ }^{*}$, and Jeffrey D. Macklis ${ }^{1,2}$ \\ ${ }^{1}$ MGH-HMS Center for Nervous System Repair, Departments of Neurosurgery and Neurology, \\ and Program in Neuroscience, Harvard Medical School; Nayef Al-Rodhan Laboratories, \\ Massachusetts General Hospital; and Department of Stem Cell and Regenerative Biology and \\ Harvard Stem Cell Institute, Harvard University
}

\begin{abstract}
Callosal projection neurons (CPN) are a diverse population of neocortical projection neurons that connect the two hemispheres of the cerebral cortex via the corpus callosum. They play key roles in high-level associative connectivity, and have been implicated in cognitive syndromes of high-level associative dysfunction, such as autism spectrum disorders. CPN evolved relatively recently compared to other cortical neuron populations, and have undergone disproportionately large expansion from mouse to human. While much is known about the anatomical trajectory of developing CPN axons, and progress has been made in identifying cellular and molecular controls over midline crossing, only recently have molecular-genetic controls been identified that specify CPN populations, and help define CPN subpopulations. In this review, we discuss development, diversity, and evolution of CPN.
\end{abstract}

\section{Introduction}

The cerebral cortex, and neocortex in particular, contains hundreds of distinct neuronal subtypes that enable it to perform incredibly complex tasks. Complexity of cognition and associative behavior expands with increased abundance and diversity of unique, yet precise connectivity among these neuronal subtypes[1]. Callosal projection neurons (CPN) play a key role in this high-level associative complexity. They are interhemispheric commissural pyramidal neurons whose myelinated axons make up the corpus callosum, the largest white matter tract in the placental mammalian brain, which connects the two cerebral hemispheres[2]. This review will discuss recent studies that have advanced our understanding of the development, diversity, and evolution of CPN.

\section{Development of the neocortex}

During neocortical development, initial populations of partially fate-restricted progenitors sequentially give rise to the wide variety of diverse mature neural cells - neurons, astroglia, and oligodendroglia[3]. The diverse neocortical neuronal population can be broadly divided into two main divisions: excitatory glutamatergic projection neurons, and inhibitory

\footnotetext{
${ }^{2}$ To whom correspondence should be addressed: Jeffrey D. Macklis, MGH-HMS Center for Nervous System Repair, Edwards 410, 50 Blossom Street, Boston, MA 02114, jeffrey_macklis@hms.harvard.edu, Phone: (617) 724-0678, Fax: (617) 726-2310.

These two authors contributed equally to this work.

Publisher's Disclaimer: This is a PDF file of an unedited manuscript that has been accepted for publication. As a service to our customers we are providing this early version of the manuscript. The manuscript will undergo copyediting, typesetting, and review of the resulting proof before it is published in its final citable form. Please note that during the production process errors may be discovered which could affect the content, and all legal disclaimers that apply to the journal pertain.
} 
GABAergic interneurons. Excitatory neocortical projection neurons arise primarily from apical and early basal intermediate progenitors in the dorsal (pallial) ventricular zone (VZ) and later from an intermediate population of basal progenitors in the subventricular zone (SVZ)[4,5], while interneurons arise from progenitors in the ventral (subpallial) ganglionic eminences[6]. The six-layered neocortex progressively forms in an inside-out fashion during embryonic development, with later-born superficial layer neurons migrating past the earlierborn deep layer neurons. Superficial neocortical layers arise primarily from later born intermediate basal progenitors of the SVZ, a type of transit amplifying progenitor generated from radial glia progenitors [7]. Neurons with the same laminar location share a common birthdate[8,9]; however, even within a layer, many anatomically and molecularly distinct neuronal subtypes coexist. Thus, substantial neuronal diversity exists both between and within layers, and laminar position is not nearly sufficient to define neuronal subtype identity and function. While broad controls governing cortical patterning are well studied, only recently have neuron subtype-specific controls begun to be elucidated[10-21].

Three broad subclasses exist within the class of excitatory cortical projection neurons corticofugal projection neurons (CFuPN), callosal projection neurons (CPN), and ipsilateral circuit connection neurons. CFuPN project away from the cortex; they respect the midline, are earlier-born, and reside in the deep layers (as is discussed later in this review, CFuPN are also evolutionarily older). CFuPN project both subcortically to deeper brain areas, and subcerebrally to brainstem and spinal cord targets. In contrast, CPN cross the midline in the brain, project interhemispherically, and function to integrate information between the two cerebral hemispheres. CPN are more abundant than CFuPN and comprise the largest class of commissural neurons in placental mammals[2]; their cell bodies principally reside in cortical layers II/III (approximately $80 \%$ in rodents), layer V (approximately $20 \%$ in rodents), and to a lesser extent layer VI.

Abnormalities of CPN are pathological. Absence of CPN connectivity in humans is associated with defects in abstract reasoning, problem solving, and generalization[22]. CPN dysgenesis is one of only a few reproducibly identified pathologies in autism spectrum disorders (ASD), with reduced corpus callosum connectivity relative to overall brain volume observed in affected individuals, often with a smaller corpus callosum[23-28]. In addition, complete or partial agenesis of the corpus callosum, as well as surgical callosotomy, result in cognitive deficits that differ depending on when in development CPN connections are lost, how much of the corpus callosum is disturbed, and the extent of secondary effects, such as aberrant connectivity[22]. The potential for identifying roles of unique populations of neurons specifically affected in diseases, such as ASD, will be enhanced by understanding neuronal diversity of projection neurons themselves within the neocortex. Molecular diversity underlies this stark anatomical and functional dichotomy between the two broad subclasses of CFuPN and CPN, as well as diversity within these subclasses.

\section{Callosal Projection Neurons}

\section{Early Development and Axon Guidance}

CPN maturation follows a set of discrete sequential steps as CPN establish correct circuitry and synaptic connections with their targets in the contralateral hemisphere. CPN are born throughout corticogenesis at the same times in development as other neurons with cell bodies residing in their same cortical layers: in the mouse, layer VI CPN are born at approximately embryonic day 12.5 (E12.5) along with corticothalamic projection neurons (CThPN); layer V CPN are born around E13.5 along with corticospinal motor neurons (CSMN); and superficial layer CPN are born from approximately E15.5 to E17.5[14,29]. As CPN are born, the two telencephalic hemispheres begin fusing, aided in part by two populations of glia - midline zipper glia and indusium griseum glia[30-33] (Figure 1a). 
While cellular and molecular mechanisms of midline fusion have not been completely identified, it is clear that if the midline is not fully fused, callosal axons have no substrate and cannot cross to the contralateral hemisphere. Many midline fusion and glial defects cause partial or complete agenesis of the corpus callosum, independent from abnormalities of CPN themselves (see Ref. [34] for review).

As the hemispheres continue to fuse, populations of glia and local neurons form the transient bridge-like subcallosal sling across the midline $[30,35,36]$. CPN send axons ventrally toward the intermediate zone guided, in part, by signals from indusium griseum glia dorsally at the midline and glial wedge and subcallosal sling populations ventrally[32,33,36]. Upon reaching the intermediate zone, callosal axons turn toward the midline to cross at the corticoseptal boundary, rather than projecting laterally as CFuPN do. Upon encountering the contralateral glial wedge, CPN axons turn dorsally and extend into the neocortex toward homotopic targets. Mechanisms of precise CPN targeting to contralateral homotopic regions are still largely unknown, but callosal axons have been shown to follow the trajectory of radial glia in the contralateral hemisphere as they extend their axons to appropriate targets[37]. During development (at E17 in mouse), axons from neurons of the cingulate cortex begin the process of midline crossing[30,38-40] and might act as pioneers for neocortical CPN[41] (Figure 1a), which begin to cross one day later[30]. First-born, deeplayer cingulate pioneers and neocortical CPN are the first of each respective population to cross the midline and, therefore, reach final targets before superficial layer CPN.

Processes of midline crossing and targeting are mediated by a large number of long-range and short-range signals that, while highly studied, are not completely known. Key molecular regulators controlling midline crossing and targeting are best studied for deep layer, earlycrossing CPN; it is not evident whether or not superficial layer, later-born CPN employ the same mechanisms. These processes have been extensively reviewed elsewhere[31,32,36,38] and we will only briefly summarize that body of work here.

Studies over the past decade have uncovered some midline crossing and targeting controls that operate at several different levels of CPN function. At the level of growth cone dynamics in callosal axons themselves, Mammalian Enabled (Mena) plays a role in actin cytoskeletal dynamics in neurons of neocortical layers II/III and V, and is required for proper formation of the corpus callosum as well as the hippocampal commissure[42]. Longrange guidance molecules, such as members of the Slit/ Robo, Wnt, and Netrin families also play active roles in axon guidance across the corpus callosum. Slits, including Slit2, are enriched along the midline, surrounding the area through which CPN axons pass; these axons express the Slit receptor Robo1 [31,43-47]. Wnts, particularly Wnt5a, are necessary for formation of all forebrain commissures, both through canonical, Frizzled3-mediated[48], and non-canonical, Receptor-like Tyrosine kinase (Ryk)-mediated[49,50], receptor transduction pathways[31]. In addition to Slits and Wnts, Netrin1 and its receptor, deleted in colorectal cancer (DCC), are also required for all forebrain commissure formation[31,5153]; however, although CPN express DCC, there is no evidence that DCC-mediated mechanisms of guidance are the same in the corpus callosum as they are for Netrin1 in commissure formation of the spinal cord[51,54]. At least some guidance roles of the subcallosal sling are mediated by Semaphorin-3C (Sema3C) attraction through the Neuropilin1 receptor on $\mathrm{CPN}[36,55]$. In addition to long-range signals, short-range, local interactors - in particular ephrins and their receptors (EphA5, EphB1, and EphrinB3) -are essential for corpus callosum formation[31,56,57]. Notably, it appears rare for guidance defects in corpus callosum formation to be callosum-specific; rather, they typically affect broader populations of commissures. 
As a broad population, CPN extend exuberant projections throughout development, with the maximal number of CPN with dual projections occurring at approximately postnatal day 8 (P8) in mice[58,59]. Dual projections are progressively refined until approximately P21, when the adult projection pattern for CPN is established; this process is thought to occur largely through activity-dependant, Hebbian mechanisms[59-62]. Interestingly, laterally located CPN that are furthest from the midline and the many signaling molecules present there, extend a bifurcated axon early in development that projects toward both the midline and the internal capsule. Only later (approximately P11 in mice) do lateral developing CPN retract axonal segments projecting to the internal capsule[63].

\section{Anatomical Diversity}

CPN extend an axon to the homotopic region of the contralateral neocortex; thus, the location of a CPN within the cortex defines the target of its callosal axon[64]. The corpus callosum is often broadly categorized in six regions from rostral to caudal, named for homotopic regions these axons connect: frontal, motor, somatosensory, auditory, temporoparietal, and visual. Callosal fibers vary in density and diameter across these regions, both in rodents and humans[2].

In addition to homotopic, interhemispheric projections extended by all CPN, subpopulations of CPN can be defined by the variety of long-range dual axonal projections they extend. Subpopulations of CPN send dual projections to contra- or ipsilateral striatum (here, referred to as CStrPNi)[65], caudally to contra- and/or ipsilateral primary somatosensory cortex (here, referred to as BPN)[58,66], or rostrally to contra- or ipsilateral frontal areas (here, referred to as FPN)[58] (Figure 1b). CPN with dual projections reside preferentially in the deep layers of the neocortex. For example, in adult mice, only about $4 \%$ of layer II/III CPN extend dual axonal projections to the frontal premotor cortex, while approximately $40 \%$ of layer V CPN do[58]. CStrPNi reside almost exclusively in layer Va[65], which supports the hypothesis that CPN with dual projections reside in evolutionarily-older deep layers, and, therefore, are more likely to have been evolutionarily co-opted from existing CFuPN. Additionally, deep layer CPN (layers V and VI) provide about $80 \%$ of the collaterals connecting primary motor cortex to primary somatosensory cortex, and some deep layer CPN have also been shown to project to secondary somatosensory cortex and the claustrum, in addition to the striatum[67]. In patients with partial agenesis of the corpus callosum, diffusion tensor imaging detects heterotopic axonal projections that are not detectable in healthy subjects[68]. It is possible that, in healthy subjects, these heterotopic projections are still present, but undetectable over overwhelming signal from intact, homotopic axons. If this is the case, there might be much more diversity of connectivity within the human corpus callosum than investigators have been able to detect with current technology.

While deep layer CPN have long-distance dual projecting axons, superficial layer CPN participate in local circuitry within cortical columns. Ipsilaterally, superficial layer CPN send collaterals to pyramidal neurons within layers II/III, and even more strongly to layer V. They also send collaterals to pyramidal and stellate neurons in layer VI. In addition to participating in column circuitry locally, superficially-located CPN also extend collaterals within the contralateral cortex, as their axons project radially into the neocortex after crossing through the corpus callosum[69]. Thus, in addition to their role in integrating two homotopic regions of the neocortical hemispheres, CPN are responsible for association and integration among different neuronal types in ipsilateral and contralateral cortical hemispheres.

Such laminar, anatomical, and connectivity diversity within the broad population of CPN demonstrate that it is not a homogenous population of projection neurons. Rather, CPN 
make up a strikingly diverse set of sub-populations requiring precise control of their neuronal diversity by a complex and interactive set of molecular-genetic controls.

\section{Molecular-Genetic Controls over CPN Development and Diversity}

Although, as discussed earlier, much progress has been made in beginning to understand the anatomical trajectory of developing CPN axons and cellular and molecular controls over midline crossing [30-32,36-38, $\left.{ }^{42}, 44-46,54,70-72\right]$, considerably less is known about molecular-genetic controls that specify CPN subtype identity and control this precise development. Because the majority of CPN reside in superficial layers, the first identified molecular controls over CPN generation and development were identified as laminarspecific genes (Table S1). In 2002, two POU-domain transcriptional regulators, Brn1 and $B r n 2$, were identified as expressed in superficial cortical layers, and as necessary for correct cortical lamination and neuronal migration[73,74]. When both Brn1 and Brn2 are deleted, superficial layer pyramidal neurons are not generated[74]. In addition, transcription factors cut-like homeobox 1 and 2 (Cuxl and $C u x 2)$ are expressed in the SVZ and selectively in superficial neocortical layers[75], with $\mathrm{Cu} 2$ 2 function being necessary for SVZ formation [76]. In 2008, the first critical molecular regulator of broad CPN specification, special ATrich sequence-binding protein 2 (SATB2), was identified and characterized as a DNAbinding transcription factor expressed in CPN. SATB2 is necessary for specification of CPN through repression of COUP-TF interacting protein 2 (CTIP2)[16,17], a transcription factor critical for CSMN axon outgrowth and fasciculation[10,11,14,77,78]. In the absence of SATB2 function, neurons that would have extended axons across the corpus callosum instead project subcortically through the internal capsule and take on some molecular characteristics of CFuPN. Identification of SATB2 as a molecular regulator of CPN identity across all layers, especially axonal connectivity through inhibition of CTIP2, significantly advanced the characterization of CPN at a molecular level. However, many interesting questions about instructive molecular signals responsible for midline crossing and precise homo- and heterotopic connections remain to be answered. Importantly, mechanisms by which this and other still-uncharacterized signals govern general CPN development are still largely unknown.

Recently, molecular-genetic controls that act specifically in subclasses of CPN have begun to be identified. The transcription factor activator enhancing binding protein 2 gamma (AP2 $\gamma$ ) acts specifically in a subset of radial glia cortical progenitors to specify SVZ intermediate progenitors and enable the switch from proliferative to neurogenic division, and to generate a specific subpopulation of superficial layer CPN in visual cortex[79]. Interestingly, while the action of AP $2 \gamma$ is highly area specific, the expression of AP $2 \gamma$ is not, suggesting an areally-restricted partner or compensatory activity. In addition, Cuxl and Cux2, previously discussed as layer-specific identifiers, regulate dendrite branching, spine development, and synapse formation specifically in layer II/III CPN[80]. These subtypespecific controls are important for understanding the diversity that exists within and is integral to the broad CPN population.

Multiple approaches have been used successfully to identify molecular controls over temporal and/or laminar stages of neocortical projection neuron development. Investigators have screened gene expression databases for transcriptional regulators expressed in relevant laminae or progenitor zones of the neocortex, or have investigated functions of guidance molecules known to play critical roles in guiding neuronal populations in other regions of the nervous system. However, in order to identify molecular controls over development of specific, individual populations of neocortical projection neurons in a more direct and unbiased manner, approaches to isolate and purify individual neuronal populations have provided substantial power and sensitivity. One approach that has proven useful for isolating distinct populations of cortical projection neurons has been to first retrogradely label them 
from their developmental axonal trajectories and final axonal targets, and then to purify them using fluorescence activated cell sorting (FACS) (Figure 2). For a variety of reasons discussed elsewhere[10,11,14], this approach was first used to label and isolate CPN[10,8183], CSMN $[10,11,84]$, corticotectal projection neurons (CTPN[10]), CThPN, CStrPN, and segmentally specific cervical or lumbar CSMN. These purified neurons were submitted to comparative microarray analysis to identify genes differentially expressed by each population at four key embryonic and postnatal developmental stages (E18.5, P3, P6, P14). Other purification methods have also proven fruitful for isolating distinct populations of cortical projection neurons $[85,86]$.

Purification of specific neuronal populations, followed by comparative gene expression analyses has not only led to the identification of genes expressed by each population at distinct stages in development, but has also enriched for critical subtype-specific molecular controls by comparing gene expression between very closely related cortical projection neuron populations. This work has already identified a set of genes that, in combination, define a progressively restricting program of molecular-genetic controls (or a "molecularlogic") over development of important populations of cortical projection neurons including CPN $[10,11,15,18,21,81,87]$. These data were collected as symmetric with regard to development of the neocortical projection neuron populations compared, and provides equivalent (but not yet functionally investigated) information regarding molecular-genetic controls over CPN development both as a broad population and in specific subpopulations[81] (Figure 3, Table S1 in supplementary material online).

CPN genes identified in this way have been analyzed based on their laminar- and sublaminar-specific distributions across different stages of maturation [81] (Figure 3, Table $\mathrm{S} 1$ in supplementary material online). Temporal information from these analyses identifies distinct molecular stages of CPN development that likely reflect known processes occurring during CPN maturation. Molecular controls expressed most highly early in CPN development (i.e.. at or before E18.5 in the mouse, such as Inhba, Btg1, Frmd4b, Epha3, and $P t n$ ) likely act during neuronal subtype specification, refinement of differentiation, migration, or initial axonal extension. Genes whose expression sharply rises and falls (i.e.. are specifically expressed only during the mid-stages of CPN development, such as Cpne4, Tmtc4, Nnmt, Cav1, Nectin-3, and Chn2) might be hypothesized to function when CPN have already crossed the midline and are extending toward their specific targets. Genes expressed specifically late in CPN development (e.g. Plexin-D1, Gfra2, TcrB, and Dkk3) might more likely function in final CPN maturation and refinement of adult connectivity.

In addition to temporal gene expression data, this work identifies differential subtypespecific laminar gene expression. A subset of the identified CPN genes appear specific to $\mathrm{CPN}$ in all layers in which $\mathrm{CPN}$ reside (i.e. layers II/III and V-VI), while others discriminate between CPN of deep layers and those of superficial layers (Figure 3). Further, several genes finely subdivide CPN within individual layers, and appear to label discrete CPN subpopulations that have not been previously described using anatomical or morphological criteria[58,81]. Interestingly, while a number of the genes expressed specifically in superficial layer CPN are expressed throughout the entirety of layers II/III, some genes are restricted to only the most superficial portion of layers II/III, while other genes are restricted to the deeper portion of superficial layers (Figure 3).

In isolation, differences in laminar expression might merely reflect major or minor birthdate differences. However, in light of being specific to CPN versus other neocortical projection neurons, and considering the existence of diverse hodological CPN subpopulations already identified, these differentially expressed genes might be hypothesized to be molecular controls and/or functional hallmarks of these previously identified subpopulations, as well as 
of novel, as-of-yet unidentified subpopulations of CPN. Molecular-genetic controls expressed in all laminae where CPN reside are more likely to control top-level, unifying properties of CPN, such as midline crossing and avoidance of corticofugal fate and connectivity. Alternatively, these broadly expressed genes might have more restricted functions due to combinatorial intersection with areally restricted binding partners and/or co-factors (such precedents exist for a number of broadly expressed cortical genes including Bhlhb5[18], Clim and Lmo4[19], and AP2 $\gamma[79])$.

Since superficial layers have undergone substantial evolutionary expansion in comparison to deep layers (as discussed in more detail below), genes with expression restricted to deep layers might reflect transcriptional changes that allowed CPN to arise from evolutionarily older CFuPN populations[15,19,88]. Deep cortical layers also contain the overwhelming majority of CPN with dual-projecting axons[58,65], so genes expressed in subpopulations of deep layer CPN might, for example, identify and control development of specific dualprojecting populations. In contrast, genes restricted to superficial layer CPN might serve as controls over development of columnar collaterals. Alternatively, CPN expressing superficial layer-restricted genes might represent novel CPN subpopulations that evolved later and are born later in cortical development than deep layer CPN. In addition, differential combinations of these genes identify even more sublaminae than are observed by examining single genes in isolation. Thus, it is evident that there is striking molecular, and likely hodological and functional, diversity within the broad population of CPN. Investigation of functions of these combinatorial molecular-genetic controls will allow for better characterization and understanding of functions and clinical relevance of these and other unique subpopulations of CPN.

\section{Evolution}

CPN and their associated axonal pathway in the corpus callosum arose relatively recently in evolution; observed first in placental mammals[2]. The corpus callosum is not unique in its ability to connect the two neocortical hemispheres, but is the only fiber tract devoted solely to integration of information from the two cortical hemispheres. In non-placental mammals, such as marsupials, the dominant interhemispheric fiber tract in the brain is the much smaller anterior commissure, consisting of interhemispheric fibers from the amygdala, olfactory tract, and temporal lobes, as well as long-distance connections from the neocortex that take a convoluted route to project to the contralateral neocortex[2]. The hippocampal commissure also plays a role, albeit much smaller than the anterior commissure, in enabling interhemispheric communication in animals lacking a corpus callosum $[2,89]$. Pre-existence of these other commissural tracts might have more readily allowed for evolution and establishment of cortical commissural projection neurons that became CPN. For example, hippocampal commissure axons, in addition to cingulate axons, might pioneer the path that neocortical CPN later follow across the corpus callosum, suggesting that these hippocampal commissural neurons might have fasciculated with emerging early CPN to enable their crossing of the midline barrier to first project across the region that is now the corpus callosum $[34,38,89]$.

The broad laminar distribution of CPN speaks not only to a broad time window of CPN generation in development from diverse progenitor populations, but also suggests preferential evolutionary expansion of this neuronal population as the cortex expanded throughout evolution. The telencephalon of sauropsids (e.g. reptiles and birds) is a threelayered structure that is evolutionarily related to layers I, V, and VI in the mammalian neocortex, but is devoid of CPN[88,90]. The evolutionarily novel cortical layers II/III, which are present in rodents, are greatly expanded in primates[88,91] (Figure 4), as is the volume of white matter in the corpus callosum[1], with over 190 million axons in the human[92]. These mammalian neocortical superficial layers arise primarily from progenitors 
of the SVZ[7], which is a distinct progenitor zone present in mammals and some sauropsids (including birds and crocodilians, but not turtles), but not in amphibians[90]. The SVZ itself has greatly expanded and diversified in primates to include two distinct regions, the inner and the outer SVZ, which generate the expanded cortical diversity, particularly in superficial layers, found in primates[91,93,94] (Figure 4). The outer SVZ contains unique selfrenewing, proliferative, radial glia-like progenitors that are distinct from those in the inner SVZ[93,94]. A number of molecular controls over SVZ populations have been identified, including T-box brain gene 2 [(Tbr2), also known as Eomes][95-97], and subventricularexpressed transcript 1 (Svet1)[7]. Given that the majority of CPN reside in the evolutionarily expanded superficial layers generated from this progressively expanded population of intermediate progenitors, it is logical to hypothesize that CPN have undergone extensive expansion throughout evolution of the cerebral cortex. Thus, compared to deep layerrestricted $\mathrm{CFuPN}, \mathrm{CPN}$ might be predicted to serve especially important roles in primate cognitive function.

Expansion of superficial neocortical layers in primate evolution far exceeds expansion of deep layers [88] . A large portion of CPN with known heterotopic long-range dualprojecting axons reside in deep neocortical layers, suggesting that deep layer CPN might have been evolutionarily co-opted from existing populations of CFuPN to project not only to subcortical targets, but also across the midline to connect and integrate the two hemispheres of the neocortex. Once the early, deep population(s) of CPN were established, their presence might have favored expansion of the neocortex, and addition of more subtypes of CPN to augment the functionally advantageous rapid and precise integration of neocortical hemispheres, perhaps first in primary visual cortex[2] or newly evolving motor cortex[89]. Interestingly, there is a large group of $\mathrm{CPN}$-enriched genes expressed in sublaminae within superficial neocortical layers in mouse[81]. In the mouse, neocortical layers II and III are not typically distinguished as distinct, but they are expanded and obviously distinct in primates. While developmental expression of these sublaminar CPN genes in primate cortex is not currently known, some (e.g. Cux2, Nectin-3, and Plxnd1) display similar expression in adult human cortex as in mouse cortex $[98,99]$. It is interesting to speculate that sublaminarly-distributed genes might serve as evolutionarily early molecular identifiers of the further expansion and specialization of superficial layers in primates.

\section{Conclusion}

Recent evidence supports a growing understanding and new appreciation of the striking diversity within the broad population of CPN. CPN are defined by their homotopically projecting axons across the corpus callosum, but some subpopulations of CPN have dualprojecting axons to long distance ipsilateral and contralateral targets, while other subpopulations participate in local column circuitry, both ipsilaterally and contralaterally. The progressive evolutionary emergence of complexity and increased diversity of connectivity in the cerebral cortex also suggests diversity in the origin of CPN. Deep-layer CPN might have arisen from evolutionarily older CFuPN [15,19], while superficial-layer CPN expanded greatly with the expansion of the SVZ in the progression from lower mammals to primates and humans. Currently, molecular-genetic controls over CPN as a broad population, and over specific subpopulations of CPN, are being discovered and functionally investigated. This work elucidating the connectivity, guidance, and molecular characteristics of CPN, both as a broad population and as distinct hodological and functional subpopulations of CPN, will greatly contribute to a better understanding of CPN and complex associative cognitive functions in which they play critical roles. More in-depth understanding of the specific functions of individual CPN subpopulations will enable more sophisticated understanding of cortical function, and diagnosis of neurological abnormalities 
involving CPN and the corpus callosum, including agenesis of the corpus callosum, ASD, and likely other syndromes of high-level dysfunction of associative connectivity.

\section{Supplementary Material}

Refer to Web version on PubMed Central for supplementary material.

\section{Acknowledgments}

We thank L. Pasquina for superb artistic assistance. This work was partially supported by the National Institutes of Health (Grants NS41590 and NS45523), the Harvard Stem Cell Institute, the Jane and Lee Seidman Fund for CNS Research, and the Emily and Robert Pearlstein Fund for Nervous System Repair (J.D.M.), with additional infrastructure support by National Institutes of Health Grant NS49553 (J.D.M). R.M.F. was partially supported by a National Science Foundation Graduate Research Fellowship.

\section{References}

1. Schoenemann PT, et al. Prefrontal white matter volume is disproportionately larger in humans than in other primates. Nat Neurosci 2005;8:242-252. [PubMed: 15665874]

2. Aboitiz F, Montiel J. One hundred million years of interhemispheric communication: the history of the corpus callosum. Braz J Med Biol Res 2003;36:409-420. [PubMed: 12700818]

3. Peters, A.; Jones, EG., editors. Cellular Components of the Cerebral Cortex. Plenum Press; 1984.

4. Kowalczyk T, et al. Intermediate neuronal progenitors (basal progenitors) produce pyramidalprojection neurons for all layers of cerebral cortex. Cereb Cortex 2009;19:2439-2450. [PubMed: 19168665]

5. Noctor SC, et al. Cortical neurons arise in symmetric and asymmetric division zones and migrate through specific phases. Nat Neurosci 2004;7:136-144. [PubMed: 14703572]

6. Hevner RF. Layer-specific markers as probes for neuron type identity in human neocortex and malformations of cortical development. J Neuropathol Exp Neurol 2007;66:101-109. [PubMed: 17278994]

7. Tarabykin V, et al. Cortical upper layer neurons derive from the subventricular zone as indicated by Svet1 gene expression. Development 2001;128:1983-1993. [PubMed: 11493521]

8. Bayer, SA.; Altman, J. Neocortical development. Raven Press; 1991.

9. Rakic P. Neurons in rhesus monkey visual cortex: systematic relation between time of origin and eventual disposition. Science 1974;183:425-427. [PubMed: 4203022]

10. Arlotta $\mathrm{P}$, et al. Neuronal subtype-specific genes that control corticospinal motor neuron development in vivo. Neuron 2005;45:207-221. [PubMed: 15664173]

11. Molyneaux BJ, et al. Fezl is required for the birth and specification of corticospinal motor neurons. Neuron 2005;47:817-831. [PubMed: 16157277]

12. Chen B, et al. Fezl regulates the differentiation and axon targeting of layer 5 subcortical projection neurons in cerebral cortex. Proc Natl Acad Sci USA 2005;102:17184-17189. [PubMed: 16284245]

13. Chen JG, et al. Zfp312 is required for subcortical axonal projections and dendritic morphology of deep-layer pyramidal neurons of the cerebral cortex. Proc Natl Acad Sci USA 2005; 102:1779217797. [PubMed: 16314561]

14. Molyneaux BJ, et al. Neuronal subtype specification in the cerebral cortex. Nat Rev Neurosci 2007;8:427-437. [PubMed: 17514196]

15. Lai T, et al. SOX5 Controls the Sequential Generation of Distinct Corticofugal Neuron Subtypes. Neuron 2008;57:232-247. [PubMed: 18215621]

16. Alcamo EA, et al. Satb2 regulates callosal projection neuron identity in the developing cerebral cortex. Neuron 2008;57:364-377. [PubMed: 18255030]

17. Britanova O, et al. Satb2 is a postmitotic determinant for upper-layer neuron specification in the neocortex. Neuron 2008;57:378-392. [PubMed: 18255031] 
18. Joshi PS, et al. Bhlhb5 regulates the postmitotic acquisition of area identities in layers II-V of the developing neocortex. Neuron 2008;60:258-272. [PubMed: 18957218]

19. Azim E, et al. Lmo4 and Clim1 progressively delineate cortical projection neuron subtypes during development. Cereb Cortex 2009;19(Suppl 1):i62-69. [PubMed: 19366868]

20. Azim E, et al. SOX6 controls dorsal-ventral progenitor parcellation and interneuron diversity during development of the neocortex. Nat Neurosci 2009;12:1238 - 1247. [PubMed: 19657336]

21. Tomassy GS, et al. Area-specific temporal control of corticospinal motor neuron differentiation by COUP-TFI. Proc Natl Acad Sci USA 2010:1-6.

22. Paul LK, et al. Agenesis of the corpus callosum: genetic, developmental and functional aspects of connectivity. Nat Rev Neurosci 2007;8:287-299. [PubMed: 17375041]

23. Minshew NJ, Williams DL. The new neurobiology of autism: cortex, connectivity, and neuronal organization. Arch Neurol 2007;64:945-950. [PubMed: 17620483]

24. Herbert MR, Kenet T. Brain abnormalities in language disorders and in autism. Pediatr Clin North Am 2007;54:563-583. vii. [PubMed: 17543910]

25. Mcalonan GM, et al. Differential effects on white-matter systems in high-functioning autism and Asperger's syndrome. Psychol Med 2009;39:1885. [PubMed: 19356262]

26. Freitag CM, et al. Total brain volume and corpus callosum size in medication-naïve adolescents and young adults with autism spectrum disorder. Biol Psychiatry 2009;66:316-319. [PubMed: 19409535]

27. Vidal CN, et al. Mapping corpus callosum deficits in autism: an index of aberrant cortical connectivity. Biol Psychiatry 2006;60:218-225. [PubMed: 16460701]

28. Egaas B, et al. Reduced size of corpus callosum in autism. Arch Neurol 1995;52:794-801. [PubMed: 7639631]

29. Angevine JB, Sidman RL. Autoradiographic study of cell migration during histogenesis of cerebral cortex in the mouse. Nature 1961;192:766-768. [PubMed: 17533671]

30. Silver J, et al. Axonal guidance during development of the great cerebral commissures: descriptive and experimental studies, in vivo, on the role of preformed glial pathways. J Comp Neurol 1982;210:10-29. [PubMed: 7130467]

31. Lindwall C, et al. Commissure formation in the mammalian forebrain. Curr Opin Neurobiol 2007;17:3-14. [PubMed: 17275286]

32. Richards LJ, et al. Mechanisms regulating the development of the corpus callosum and its agenesis in mouse and human. Clin Genet 2004;66:276-289. [PubMed: 15355427]

33. Shu T, et al. Development of midline glial populations at the corticoseptal boundary. J Neurobiol 2003;57:81-94. [PubMed: 12973830]

34. Donahoo ALS, Richards LJ. Understanding the Mechanisms of Callosal Development Through the Use of Transgenic Mouse Models. Semin Pediatr Neurol 2009;16:127-142. [PubMed: 19778710]

35. Shu T, et al. The glial sling is a migratory population of developing neurons. Development 2003;130:2929-2937. [PubMed: 12756176]

36. Niquille $\mathrm{M}$, et al. Transient neuronal populations are required to guide callosal axons: a role for semaphorin 3C. PLoS Biol 2009;7:e1000230. [PubMed: 19859539]

37. Norris CR, Kalil K. Guidance of callosal axons by radial glia in the developing cerebral cortex. J Neurosci 1991;11:3481-3492. [PubMed: 1941093]

38. Rash BG, Richards LJ. A role for cingulate pioneering axons in the development of the corpus callosum. J Comp Neurol 2001;434:147-157. [PubMed: 11331522]

39. Koester SE, O'Leary DD. Axons of early generated neurons in cingulate cortex pioneer the corpus callosum. J Neurosci 1994;14:6608-6620. [PubMed: 7965064]

40. Ozaki HS, Wahlsten D. Timing and origin of the first cortical axons to project through the corpus callosum and the subsequent emergence of callosal projection cells in mouse. J Comp Neurol 1998;400:197-206. [PubMed: 9766399]

41. Piper M, et al. Neuropilin 1-Sema Signaling Regulates Crossing of Cingulate Pioneering Axons during Development of the Corpus Callosum. Cerebral Cortex 2009;11

42. Lanier LM, et al. Mena is required for neurulation and commissure formation. Neuron 1999;22:313-325. [PubMed: 10069337] 
43. Shu T, et al. Slit2 guides both precrossing and postcrossing callosal axons at the midline in vivo. J Neurosci 2003;23:8176-8184. [PubMed: 12954881]

44. López-Bendito G, et al. Robo1 and Robo2 cooperate to control the guidance of major axonal tracts in the mammalian forebrain. J Neurosci 2007;27:3395-3407. [PubMed: 17392456]

45. Andrews W, et al. Robo1 regulates the development of major axon tracts and interneuron migration in the forebrain. Development 2006;133:2243-2252. [PubMed: 16690755]

46. Sundaresan V, et al. Dynamic expression patterns of Robo (Robo1 and Robo2) in the developing murine central nervous system. J Comp Neurol 2004;468:467-481. [PubMed: 14689480]

47. Bagri A, et al. Slit proteins prevent midline crossing and determine the dorsoventral position of major axonal pathways in the mammalian forebrain. Neuron 2002;33:233-248. [PubMed: 11804571]

48. Wang Y, et al. Axonal growth and guidance defects in Frizzled3 knock-out mice: a comparison of diffusion tensor magnetic resonance imaging, neurofilament staining, and genetically directed cell labeling. J Neurosci 2006;26:355-364. [PubMed: 16407530]

49. Keeble TR, et al. The Wnt receptor Ryk is required for Wnt5a-mediated axon guidance on the contralateral side of the corpus callosum. J Neurosci 2006;26:5840-5848. [PubMed: 16723543]

50. Li L, et al. Wnt5a Induces Simultaneous Cortical Axon Outgrowth and Repulsive Axon Guidance through Distinct Signaling Mechanisms. Journal of Neuroscience 2009;29:5873-5883. [PubMed: 19420254]

51. Serafini T, et al. Netrin-1 is required for commissural axon guidance in the developing vertebrate nervous system. Cell 1996;87:1001-1014. [PubMed: 8978605]

52. Ren T, et al. Diffusion tensor magnetic resonance imaging and tract-tracing analysis of Probst bundle structure in Netrin1- and DCC-deficient mice. J Neurosci 2007;27:10345-10349. [PubMed: 17898206]

53. Fazeli A, et al. Phenotype of mice lacking functional Deleted in colorectal cancer (Dcc) gene. Nature 1997;386:796-804. [PubMed: 9126737]

54. Shu T, et al. Expression of the netrin-1 receptor, deleted in colorectal cancer (DCC), is largely confined to projecting neurons in the developing forebrain. J Comp Neurol 2000;416:201-212. [PubMed: 10581466]

55. Gu C, et al. Neuropilin-1 conveys semaphorin and VEGF signaling during neural and cardiovascular development. Dev Cell 2003;5:45-57. [PubMed: 12852851]

56. Mendes SW, et al. Multiple Eph receptors and B-class ephrins regulate midline crossing of corpus callosum fibers in the developing mouse forebrain. J Neurosci 2006;26:882-892. [PubMed: $16421308]$

57. $\mathrm{Hu} \mathrm{Z}$, et al. Corpus callosum deficiency in transgenic mice expressing a truncated ephrin-A receptor. J Neurosci 2003;23:10963-10970. [PubMed: 14645492]

58. Mitchell BD, Macklis JD. Large-scale maintenance of dual projections by callosal and frontal cortical projection neurons in adult mice. J Comp Neurol 2005;482:17-32. [PubMed: 15612019]

59. Innocenti GM, Price DJ. Exuberance in the development of cortical networks. Nat Rev Neurosci 2005;6:955-965. [PubMed: 16288299]

60. Wang C, et al. Activity-Dependent Development of Callosal Projections in the Somatosensory Cortex. Journal of Neuroscience 2007;27:11334-11342. [PubMed: 17942728]

61. Mizuno H, et al. Evidence for activity-dependent cortical wiring: formation of interhemispheric connections in neonatal mouse visual cortex requires projection neuron activity. J Neurosci 2007;27:6760-6770. [PubMed: 17581963]

62. Mizuno H, et al. Pre-synaptic and post-synaptic neuronal activity supports the axon development of callosal projection neurons during different post-natal periods in the mouse cerebral cortex. Eur J Neurosci 31:410-424. [PubMed: 20105242]

63. Garcez PP, et al. Axons of callosal neurons bifurcate transiently at the white matter before consolidating an interhemispheric projection. Eur J Neurosci 2007;25:1384-1394. [PubMed: 17425565]

64. Yorke CH Jr, Caviness VS Jr. Interhemispheric neocortical connections of the corpus callosum in the normal mouse: a study based on anterograde and retrograde methods. J Comp Neurol 1975;164:233-245. [PubMed: 1184784] 
65. Wilson CJ. Morphology and synaptic connections of crossed corticostriatal neurons in the rat. J Comp Neurol 1987;263:567-580. [PubMed: 2822779]

66. Cauller LJ, et al. Backward cortical projections to primary somatosensory cortex in rats extend long horizontal axons in layer I. J Comp Neurol 1998;390:297-310. [PubMed: 9453672]

67. Veinante P, Deschenes M. Single-cell study of motor cortex projections to the barrel field in rats. J Comp Neurol 2003;464:98-103. [PubMed: 12866130]

68. Wahl M, et al. Variability of Homotopic and Heterotopic Callosal Connectivity in Partial Agenesis of the Corpus Callosum: A 3T Diffusion Tensor Imaging and Q-Ball Tractography Study. American Journal of Neuroradiology 2008;30:282-289. [PubMed: 19001538]

69. Petreanu L, et al. Channelrhodopsin-2-assisted circuit mapping of long-range callosal projections. Nat Neurosci 2007;10:663-668. [PubMed: 17435752]

70. Plachez C, et al. Nuclear factor I gene expression in the developing forebrain. J Comp Neurol 2008;508:385-401. [PubMed: 18335562]

71. Smith KM, et al. Midline radial glia translocation and corpus callosum formation require FGF signaling. Nat Neurosci 2006;9:787-797. [PubMed: 16715082]

72. Tole S, et al. Development of midline cell types and commissural axon tracts requires Fgfr1 in the cerebrum. Dev Biol 2006;289:141-151. [PubMed: 16309667]

73. McEvilly RJ, et al. Transcriptional regulation of cortical neuron migration by POU domain factors. Science 2002;295:1528-1532. [PubMed: 11859196]

74. Sugitani Y, et al. Brn-1 and Brn-2 share crucial roles in the production and positioning of mouse neocortical neurons. Genes Dev 2002;16:1760-1765. [PubMed: 12130536]

75. Nieto M, et al. Expression of Cux-1 and Cux-2 in the subventricular zone and upper layers II-IV of the cerebral cortex. J Comp Neurol 2004;479:168-180. [PubMed: 15452856]

76. Cubelos B, et al. Cux-2 Controls the Proliferation of Neuronal Intermediate Precursors of the Cortical Subventricular Zone. Cerebral Cortex 2007;18:1758-1770. [PubMed: 18033766]

77. Chen B, et al. Fezl regulates the differentiation and axon targeting of layer 5 subcortical projection neurons in cerebral cortex. Proc Natl Acad Sci U S A 2005;102:17184-17189. [PubMed: 16284245]

78. Chen JG, et al. Zfp312 is required for subcortical axonal projections and dendritic morphology of deep-layer pyramidal neurons of the cerebral cortex. Proc Natl Acad Sci U S A 2005;102:1779217797. [PubMed: 16314561]

79. Pinto L, et al. AP2 $\gamma$ regulates basal progenitor fate in a region- and layer-specific manner in the developing cortex. Nat Neurosci 2009;12:1229-1237. [PubMed: 19749747]

80. Cubelos B, et al. Cux 1 and Cux 2 regulate dendritic branching, spine morphology, and synapses of the upper layer neurons of the cortex. Neuron 2010;66:523-535. [PubMed: 20510857]

81. Molyneaux BJ, et al. Novel Subtype-specific Genes Identify Distinct Subpopulations of Callosal Projection Neurons. J Neurosci 2009;29:12343-12354. [PubMed: 19793993]

82. Catapano LA, et al. Stage-specific and opposing roles of BDNF, NT-3 and bFGF in differentiation of purified callosal projection neurons toward cellular repair of complex circuitry. Eur J Neurosci 2004;19:2421-2434. [PubMed: 15128396]

83. Catapano LA, et al. Specific neurotrophic factors support the survival of cortical projection neurons at distinct stages of development. J Neurosci 2001;21:8863-8872. [PubMed: 11698598]

84. Ozdinler PH, Macklis JD. IGF-I specifically enhances axon outgrowth of corticospinal motor neurons. Nat Neurosci 2006;9:1371-1381. [PubMed: 17057708]

85. Dugas J, et al. A Novel Purification Method for CNS Projection Neurons Leads to the Identification of Brain Vascular Cells as a Source of Trophic Support for Corticospinal Motor Neurons. Journal of Neuroscience 2008;28:8294-8305. [PubMed: 18701692]

86. Barres BA, et al. Immunological, morphological, and electrophysiological variation among retinal ganglion cells purified by panning. Neuron 1988;1:791-803. [PubMed: 2908449]

87. Arlotta $\mathrm{P}$, et al. Ctip2 controls the differentiation of medium spiny neurons and the establishment of the cellular architecture of the striatum. J Neurosci 2008;28:622-632. [PubMed: 18199763]

88. Molnar Z, et al. Comparative aspects of cerebral cortical development. Eur J Neurosci 2006;23:921-934. [PubMed: 16519657] 
89. Mihrshahi R. The corpus callosum as an evolutionary innovation. J Exp Zool B Mol Dev Evol 2006;306:8-17. [PubMed: 16116611]

90. Charvet CJ, et al. Phylogeny of the telencephalic subventricular zone in sauropsids: evidence for the sequential evolution of pallial and subpallial subventricular zones. Brain Behav Evol 2009;73:285-294. [PubMed: 19641308]

91. Smart IH, et al. Unique morphological features of the proliferative zones and postmitotic compartments of the neural epithelium giving rise to striate and extrastriate cortex in the monkey. Cereb Cortex 2002;12:37-53. [PubMed: 11734531]

92. Tomasch J. Size, distribution, and number of fibres in the human corpus callosum. Anat Rec 1954;119:119-135. [PubMed: 13181005]

93. Fietz SA, et al. OSVZ progenitors of human and ferret neocortex are epithelial-like and expand by integrin signaling. Nat Neurosci 2010;13:690-699. [PubMed: 20436478]

94. Hansen DV, et al. Neurogenic radial glia in the outer subventricular zone of human neocortex. Nature 2010;464:554-561. [PubMed: 20154730]

95. Baala L, et al. Homozygous silencing of T-box transcription factor EOMES leads to microcephaly with polymicrogyria and corpus callosum agenesis. Nat Genet 2007;39:454-456. [PubMed: 17353897]

96. Arnold SJ, et al. The T-box transcription factor Eomes/Tbr2 regulates neurogenesis in the cortical subventricular zone. Genes Dev 2008;22:2479-2484. [PubMed: 18794345]

97. Sessa A, et al. Tbr2 directs conversion of radial glia into basal precursors and guides neuronal amplification by indirect neurogenesis in the developing neocortex. Neuron 2008;60:56-69. [PubMed: 18940588]

98. Arion D, et al. Molecular markers distinguishing supragranular and infragranular layers in the human prefrontal cortex. Eur J Neurosci 2007;25:1843-1854. [PubMed: 17432970]

99. Allen-Institute-for-Brain-Science (C2009). Allen Human Cortex Study [Internet]. Available from: http://humancortex.alleninstitute.org

100. Allen-Institute-for-Brain-Science (C2009). Allen Developing Mouse Brain Atlas [Internet]. Available from: http://developingmouse.brain-map.org 

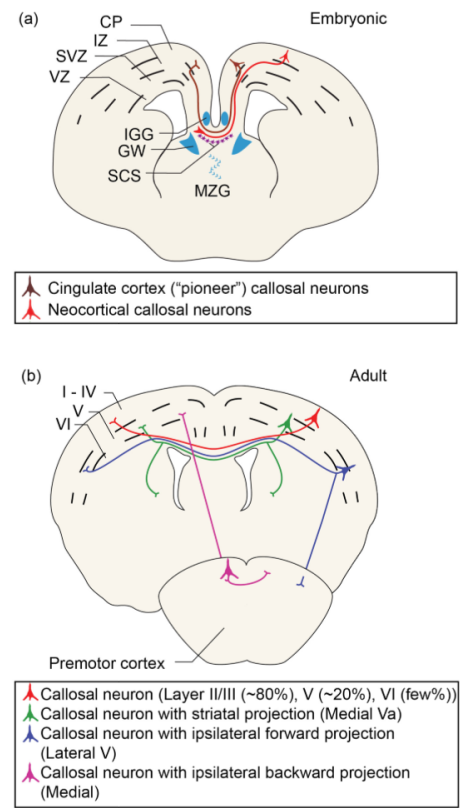

Figure 1. CPN development and diversity

(a) During development, callosal axons (red) turn toward the midline. Multiple glial populations (blue) and mixed neuronal/glial populations (purple) play critical roles in CPN axon guidance and midline crossing. Pioneering axons (brown) from neurons of the cingulate cortex begin the process of midline crossing. This schematic represents processes that occur across multiple embryonic times during mouse CPN development. Abbreviations: $\mathrm{CP}$, cortical plate; IZ, intermediate zone; SVZ, subventricular zone; VZ, ventricular zone; IGG, indusium griseum glia; GW, glial wedge; SCS, subcallosal sling; MZG, midline zipper glia. (b) At least four major types of adult CPN can be classified based on projection patterns. These include: single projections to the contralateral cortex (red); dual projections to the contralateral cortex and ipsilateral or contralateral striatum (green); dual projections to the contralateral cortex and ipsilateral premotor cortex (blue); or dual projections to the contralateral cortex and ipsilateral sensorimotor cortex (purple). 


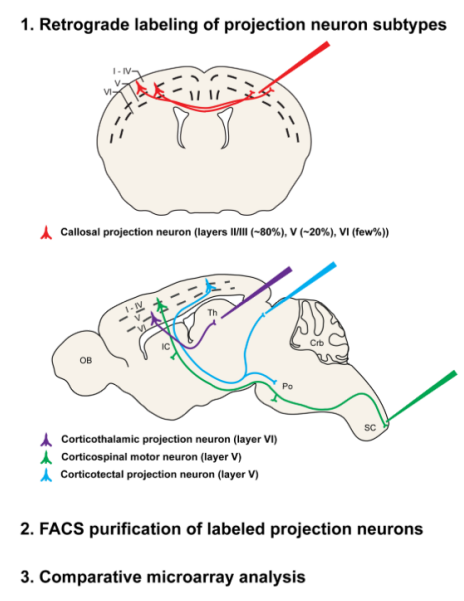

Figure 2.

Schematic representation of an experimental approach used to identify CPN-specific genes. CPN (red), corticothalamic projection neurons (purple), corticospinal motor neurons (green), and corticotectal projection neurons (blue) were retrogradely labeled at distinct stages of development from the contralateral hemisphere, the thalamus, the spinal cord, and the superior colliculus, respectively. Labeled neurons were dissociated, purified using fluorescence activated cell sorting (FACS), and followed by comparative microarray genetic expression analysis[10,14,81-84]. Adapted, with permission, from Ref. [81]. 

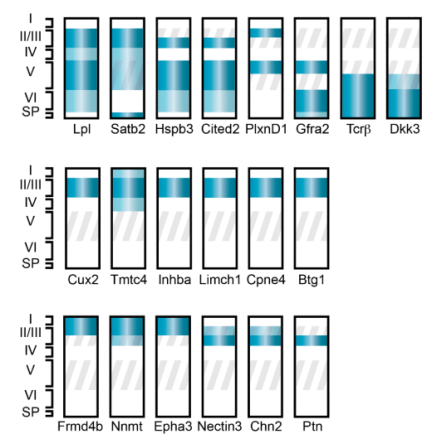

\section{Figure 3. Spatially-restricted genes identify novel CPN subpopulations}

Schematic representation of neocortical layers depicting laminar-specific expression of 20 selected, representative genes expressed by early postnatal CPN within the neocortex [81]. Dark and light blue bands indicate high and low levels of expression, respectively. Grey oblique stripes demarcate layers in which CPN reside. Most of these genes have dynamic patterns of expression through development; therefore, developmental stage must be considered when using these genes to identify specific populations of CPN. Representative genes are depicted with multiple patterns of laminar expression: most cortical layers; and deep cortical layers only (top row); superficial cortical layers only (middle row); and subdivisions of superficial layers (bottom row). See text and Table S1 in supplementary material online for more detailed expression and references. Abbreviations: roman numerals indicate neocortical layers (I-VI); SP, subplate. 
(a) Mouse

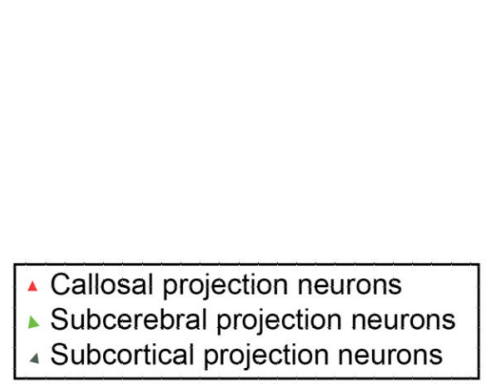

(b) Macaque

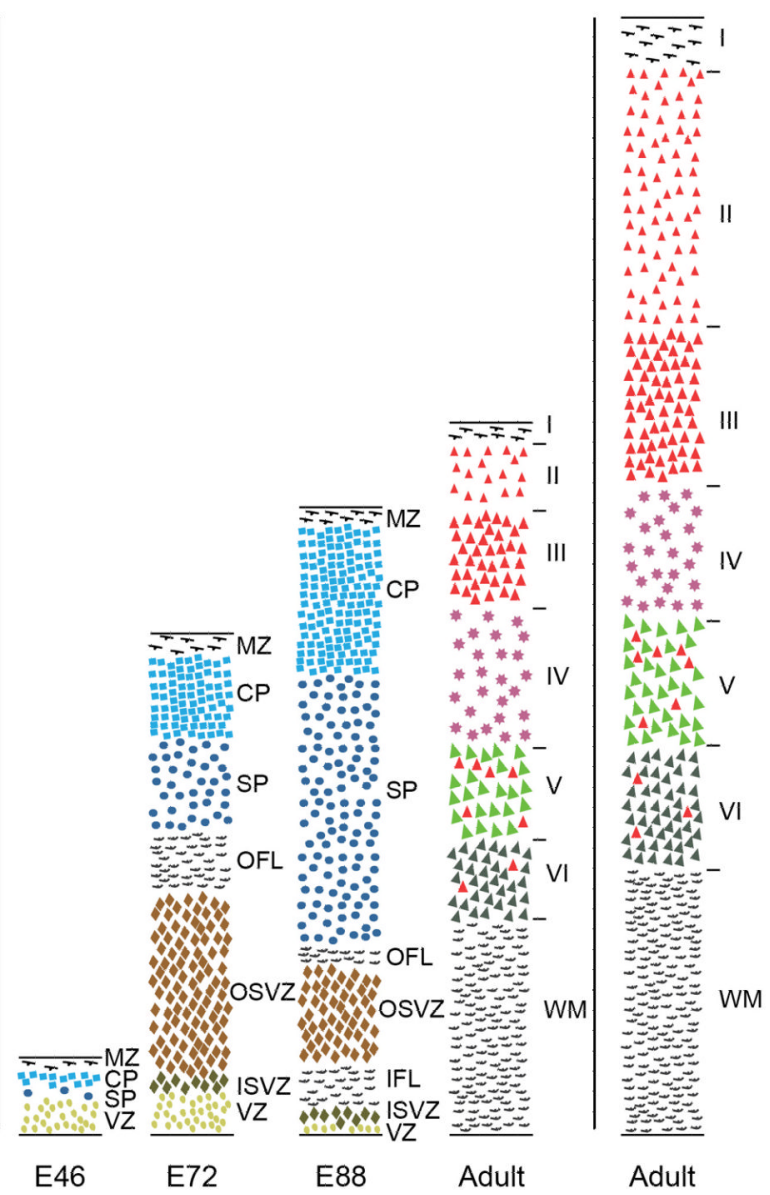

Figure 4. Comparison of developing and adult mammalian neocortex of mouse, macaque, and human shows correlations between SVZ expansion, superficial neocortical layer expansion, and white matter expansion

Schematic comparison of histological sections of (a) developing and adult mouse, (b) developing and adult rhesus macaque monkey, and (c) adult human neocortex. Adult crosssections are from visual cortex. The thicknesses are represented relative to a common scale. Callosal projection neurons (red) reside mostly in layers II/III $(\sim 80 \%), \mathrm{V}(\sim 20 \%)$, and VI (few \%) in the adult neocotex. There is a strong correlation between the expansion of the SVZ and the expansion of the superficial layer thickness and neocortical white matter. Abbreviations: E, embryonic; PP, preplate; VZ, ventricular zone; MZ, marginal zone; CP, cortical plate; SP, subplate; SVZ, subventricular zone; OSVZ, outer SVZ; ISVZ, inner SVZ; OFL, outer fibrous layer; IFL, inner fibrous layer; WM, white matter; Roman numerals denote neocortical layers (IVI). Adapted and expanded with permission from Ref. [91] (macaque) with data from Ref [100] (mouse), and Refs [1,99] (human). 